\title{
The Spectral Characteristics of Ne-Ar Gas Mixtures in a Positive Column of a Discharge Tube with and without Superimposed RF Powers
}

\author{
Yasufumi OHSONE, Teruo KANEDA \\ Department of Electronic Engineering, Faculty of Engineering, Tokyo Denki University. \\ Tokyo Denki University, \\ 2-2 Kanda Nishiki-cho, Chiyoda-ku, Tokyo, Japan, 101.
}

\begin{abstract}
The spectral intensities emitted from a positive column in a rectangular discharge tube were measured as functions of the gas pressure, mixing ratio of argon/neon, and with and without superimposed RF powers. The tube used for measurements has a rectangular profile of $2 \times 4 \mathrm{~cm}$. The discharge current was $20 \mathrm{~mA}$. The gas pressure was varied from 0.2 to 10 Torr. The superimposed RF power having a frequency of $13.56 \mathrm{MHz}$ varied from 0 to $30 \mathrm{~W}$. The experimental results were discussed qualitatively.
\end{abstract}

\section{Introduction}

Rectangular shaped discharge tubes have been studied by numerous authors ${ }^{(1)-(5)}$. Recently, the positive column in the rectangular tube has received increased attention as a result of recent developments in gaseous lasers ${ }^{(6)}$, luminance plasma used in the liquid crystal displays, plasma enhanced chemical deposition, and etching techniques in semiconductor processes ${ }^{(7)}$.

The plasma density profiles of the positive column in a rectangular discharge tube have been theoretically analyzed by Chang ${ }^{(1)}$ by expanding the ambipolar diffusion theory originally proposed by Schottky ${ }^{(8)}$. Chang et al. ${ }^{(1)}$ have also theoretically determined the electron temperature in such a tube by using ambipolar diffusion theory. Recently, Kaneda ${ }^{(9)}$ measured these plasma parameters for pure gases in a rectangular discharge tube, while also studying the optical characteristics in the discharge tube under an A.C. magnetic field $^{(10)}$. Furthermore, Kaneda ${ }^{(1)}$ examined the light intensity profiles by the addition of oxygen to helium.

On the other hand, the spectral characteristics of rare gas mixture in a cylindrical capillary discharge tube have been measured by Kaneda ${ }^{(12)}$. For these gas mixtures, it was observed that the spectral characteristics were greatly influenced by the discharge conditions such as the tube radius, discharge current, gas pressure, and gas mixing ratios. The spectral characteristics of gases in cylindrical tubes have long been studied along with the development of lamps.

However, there have been few theoretical and experimental studies concerning mixtures of rare gases in rectangular discharge tubes. Thus, it is of importance to experimentally investigate these phenomena since their fundamental optical characteristics have not been previously measured.

In this report, the spectral characteristics of a positive column in a rectangular discharge tube are measured under various discharge conditions. The gases used in the experiment are mixtures of Ne-Ar. The results of the measurements are also qualitatively discussed.

\section{Experimental procedure}

The rectangular discharge tube, made of Pyrex glass used in the present experiments is schematically illustrated in Fig. 1. The tube has two electrodes and a rectangular plasma length of $9 \mathrm{~cm}$. The electrodes consist of a hollow cylindrical cathode of stainless steel and a disc anode composed of nickel. The cathode is $70 \mathrm{~mm}$ in length and $20 \mathrm{~mm}$ in diameter. The rectangular profile of the positive column is $2 \times 4 \mathrm{~cm}$. The rectangular discharge tube was used because the RF electrodes can be easily attached to the discharge tube and the RF powers act effectively to the plasma.

The discharge tube was evacuated to $10^{-6}$ Torr by use of an oil diffusion pump. The absorbed gases on the wall and electrodes were removed by usual baking processes. The gases used in the measurements were neon, argon and mixtures of these two gases. The purity of both the neon and argon was $99.99 \%$.

The discharge current I was $20 \mathrm{~mA}$ while the gas pressure 
$p$ could be varied from 0.2 to 10 Torr, and was measured by a BARATRON gauge. The RF power having a frequency of $13.56 \mathrm{MHz}$ varied from 0 to $30 \mathrm{~W}$.

The schematic diagram illustrating the measurement procedure of the spectral characteristics is shown in Fig.2. As shown in this figure, the $R F$ electric field of $13.56 \mathrm{MHz}$ was applied perpendicular to the positive column axis over the length of $8 \mathrm{~cm}$. The gap between the RF electrodes was $3 \mathrm{~cm}$. The RF electric field was provided by an $R F$ power supply through a RF matching box. The value of SWR (Standing Wave Ratio) was set manually to be 1.0 , which corresponds to the no reflecting wave, by arranging the matching box. Therefore, RF power can be supplied to discharge tube with small leakage ${ }^{(13\rangle-(14)}$.

The radiation produced in the positive column of the discharge tube was guided to a motor driven monochrometer by a glass fiber where it was enhanced by a photomultiplier, as shown in the figure. The output voltage of the photo-

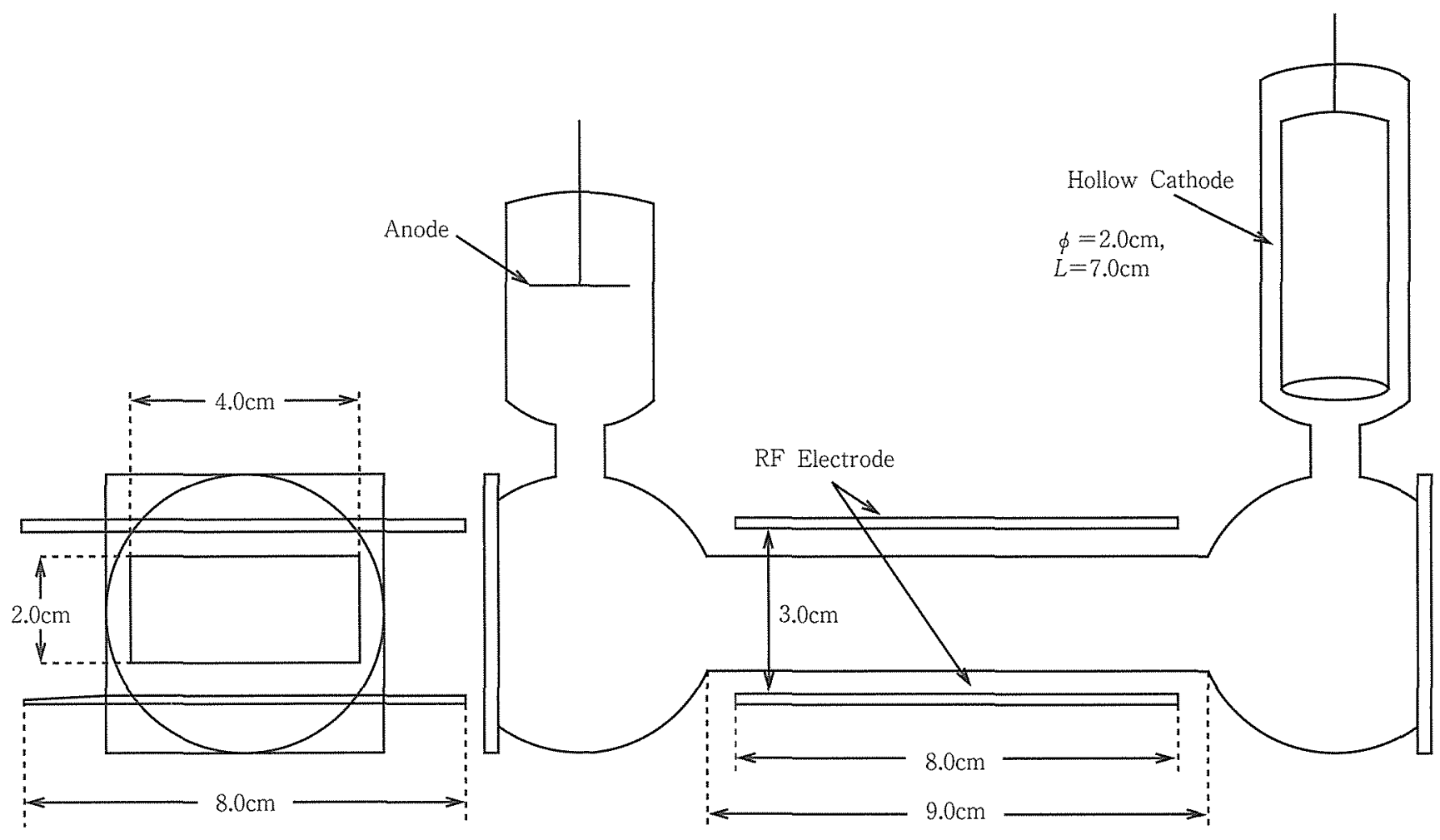

Fig. 1: Schematic diagram of the rectangular discharge tube.

HV Regurater
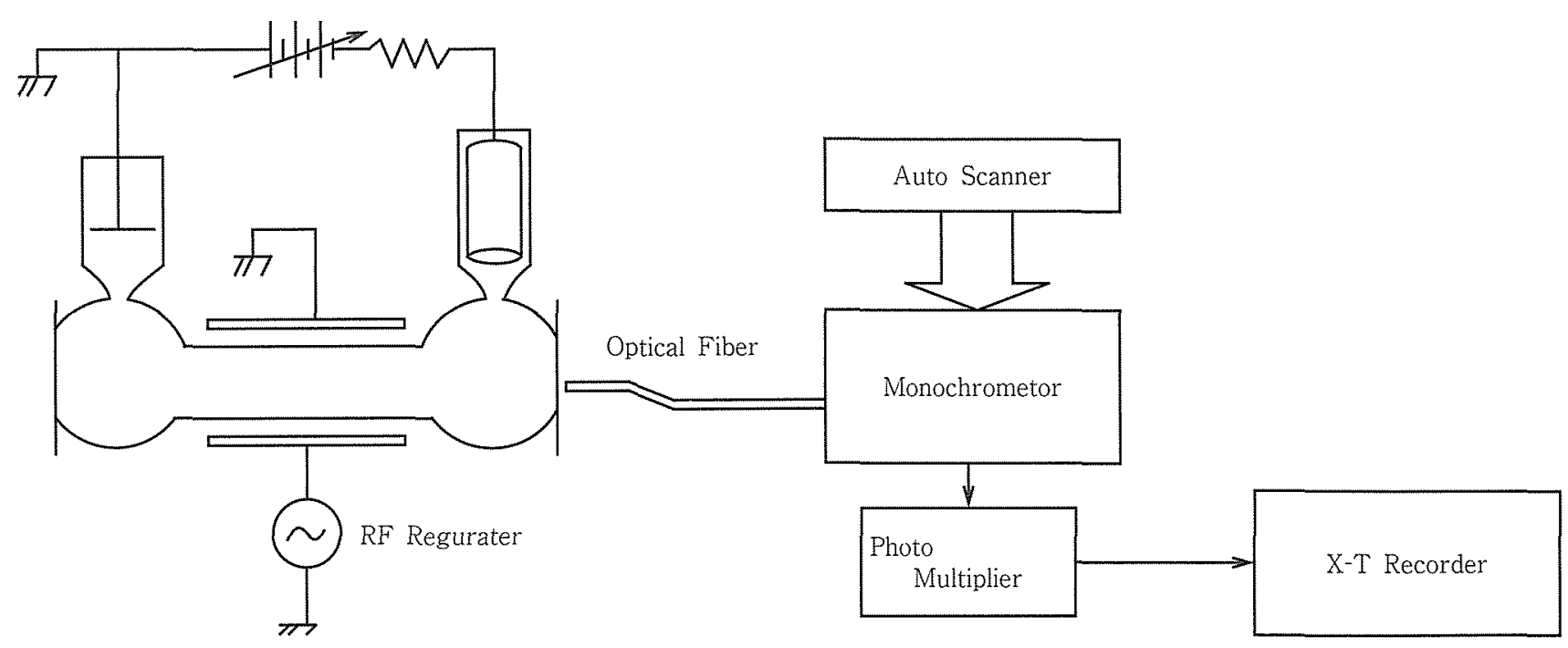

Fig. 2: Schematic of the apparatus used for the measurement of the spectral characteristics 
multiplier, as shown in the figure. The output voltage of the photomultiplier was recorded by a graphic recorder. The spectral intensity of each spectral wavelength through the optical measurement system was corrected by a standard lamp.

\section{Experimental results}

The spectral intensities of various argon/neon gas mixtures are shown in Fig. 3, where no RF power was superimposed on the discharge tube. In Fig. 3 (a), the normalized relative spectral intensities for various wavelengths of pure neon in the positive column of rectangular discharge tube are shown as functions of gas pressure. The spectral lines can be divided into two groups by the pressure dependency. The first group consists of wavelengths $\lambda=$ 5852 and $4885(\AA)$ lines. Overall, the spectral intensities of these lines decrease with increasing gas pressure. The second group is composed of wavelengths $\lambda=7032,6402$, 6505,6678 and $6148(\AA)$.In this group, the spectral intensities of the lines increase with increasing gas pressure, become saturated after the gas pressure attains a value of about 2.0 Torr, and then decrease with increasing gas pressure.

In Fig. 3(b), the normalized spectral intensities are shown when $1 \%$ of argon is mixed with the neon gas. As shown in this figure, almost all the neon lines decrease with increasing gas pressure. On the contrary, almost all the argon liens increase, attain maximum values and then decrease with increasing gas pressure. The strong line of $\lambda=7032(\AA)$, which was observed in the pure neon discharge, disappears and a strong neon line $\lambda=6402(\AA)$, which previously increased with increasing gas pressure in pure neon. decreases as the gas pressure increases.

Figure $3(\mathrm{c})$ is the same as Fig. 3 (b) except that $3 \%$ of argon is mixed with the neon gas. It is shown that the intensities of almost all the neon lines decrease with increasing gas pressure. The intensities of the argon lines are larger compared with those of the neon lines. It is also found that these argon lines increase, attain maximum values, and then decrease with increasing gas pressure.

In Figs. 3(d), and 3(e), the normalized spectral intensities for various wavelengths are shown as functions of gas pressure when argon of $5 \%$ and $10 \%$ are mixed with neon gas, respectively. As shown in these figures, the characteristics of the maximum of the spectral lines become clearer with only small differences found between the two mixtures. Moreover, it is observed that the normalized spectral intensities of various wavelengths of argon decrease with increasing the gas pressure over $p=2.0$ Torr. While the normalized spectral intensity of the neon wavelength of $6402(\AA)$ rises abruptly when the gas pressure increases over 7 Torr.

Figure $3(\mathrm{f})$ displays the normalized spectral intensities when argon of $20 \%$ is mixed with neon gas. Here, all the neon spectral lines are of negligible intensity, while almost all lines that exhibit an intensity are argon spectral lines. Moreover, it is found that the argon wavelength of 7504

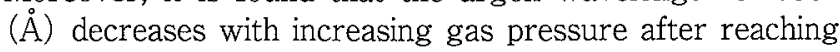
a peak in spectral intensity. Furthermore, it is noted that the lines of $\lambda=7635$ and $7948(\AA)$ exhibit no maximum characteristics.
The normalized spectral intensities for various wavelengths of pure argon are shown in Fig. $3(\mathrm{~g})$ as functions of gas pressure. Almost all lines of the spectrum decrease with increasing gas pressure. It is found that the wavelength of $7635(\AA)$ is a strong line, and its intensity increases with increasing gas pressure, similar to its behavior in Fig. $3(\mathrm{f})$.

Figure 4 examines the spectral intensities as a function of the gas mixing ratio (argon/neon +argon $\mathrm{x} 100$ ) under conditions of no RF power.

In Fig. 4 (a), the normalized spectral intensities with the pressure held at $p=0.2$ Torr are shown as a function of the gas mixing ratio. The neon spectral lines for the wavelengths $\lambda=6142,6505$, and $7032(\AA)$, which are strong in pure neon, do not appear when a small amount of argon $(1 \%)$ is added to neon. The spectral intensities for the neon wavelengths $\lambda=5852$ and $6402(\AA)$ slightly increased by the addition of $10 \%$ argon and then gradually decreased as the mixing ratio of argon increased. The spectral line of neon for the wavelength $\lambda=4885(\AA)$ decreases sharply with the addition of $1 \%$ argon and then gradually decreases as the mixing ratio of argon increases. On the other hand, the spectral intensities of argon for the wavelengths $\lambda=$ 7504,7635 , and $7948(\AA)$, which are comparably strong in pure argon, have similar intensities as those of the neon spectrum at a $1 \%$ argon mixing. These lines display a considerable increase at a mixing ratio of $3 \%$ argon, and then decrease when the mixing ratio reaches $5 \%$. They again increase as the mixing ratio of argon is further increased.

Figure 4 (b) is the same as in Fig. 4 (a) except under conditions of $p=1.0$ Torr. Here, the spectral intensities for the wavelengths $\lambda=6143,5605$, and 7032 ( $\AA$ ) of neon do not appear with the addition of $1 \%$ argon, similar to that observed for $p=0.2$ Torr. The neon spectral intensities for wavelengths $\lambda=5852,6402$, and $6678(\AA)$ decrease abruptly with the increase in the argon ratio and then gradually decreases as the ratio is further increased. However, the neon spectral intensity of wavelength $4885 \AA$ increases at the argon mixing ratio of $3 \%$ and then gradually decreases when the mixing ratio increase. On the other hand, the argon spectral intensities for the wavelengths $\lambda=7504,7635$, and 7948 ( $\AA$ ) become larger than almost all neon line when only $1 \%$ argon is added to the neon. When the mixing ratio of argon attains $3 \%$, these spectral lines become almost dominant. Similar to the characteristics for the condition of $p=0.2$ Torr, the intensities of these lines suddenly decrease at the mixing ratio of $5 \%$, and then increase as mixing ratio is further increased.

Figure 4 (c) exhibits the spectral intensities at a pressure of $p=4.0$ Torr as a function of the gas mixture. At this pressure, the spectral intensities for wavelengths $\lambda=4885$, 6143 , and $7032(\AA)$ of neon are not found with the addition of $1 \%$ argon. Furthermore, the neon spectral intensities for wavelengths $\lambda=5852,6402$, and $6678(\AA)$, which are strong lines in pure neon, exhibit sudden decrease when $1 \%$ argon is added and then disappear when the mixing ratio of argon reaches $3 \%$. The lines of argon play a dominant role when $1 \%$ of argon is mixed with the neon gas. As observed for a pressure of $p=1.0$ Torr, the spectral inten- 


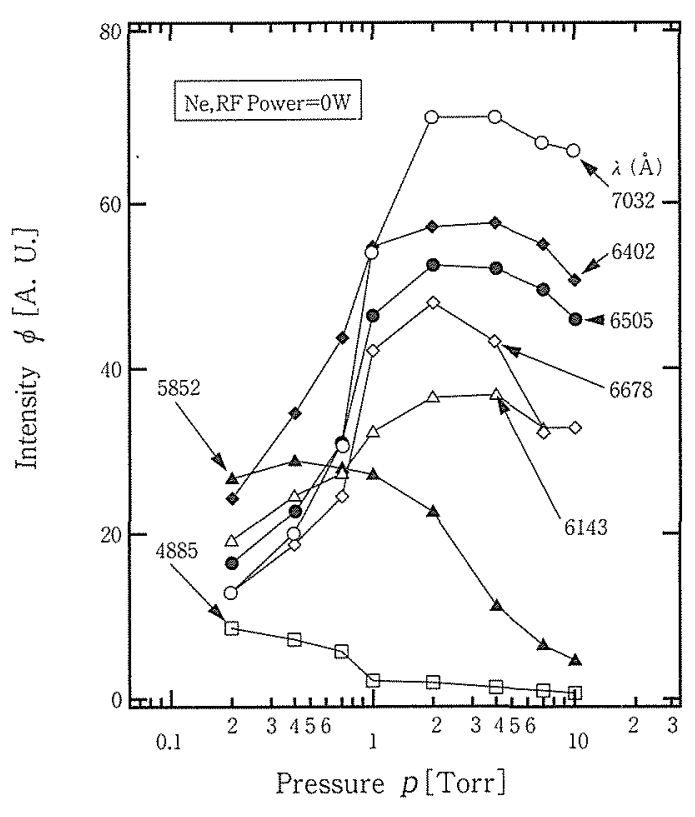

Fig. 3 (a)

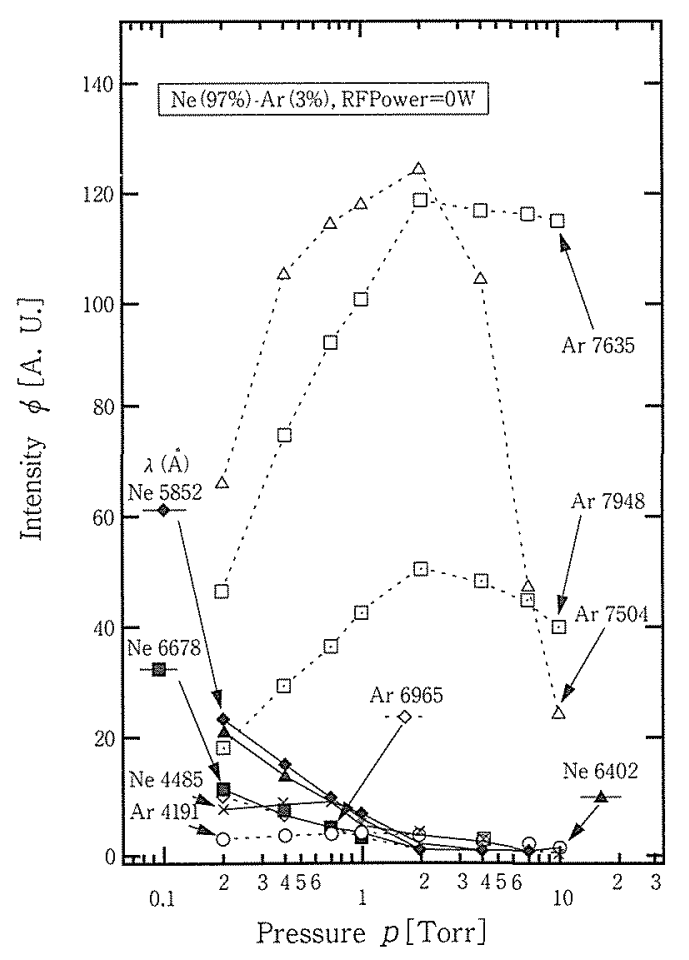

Fig. $3(\mathrm{c})$

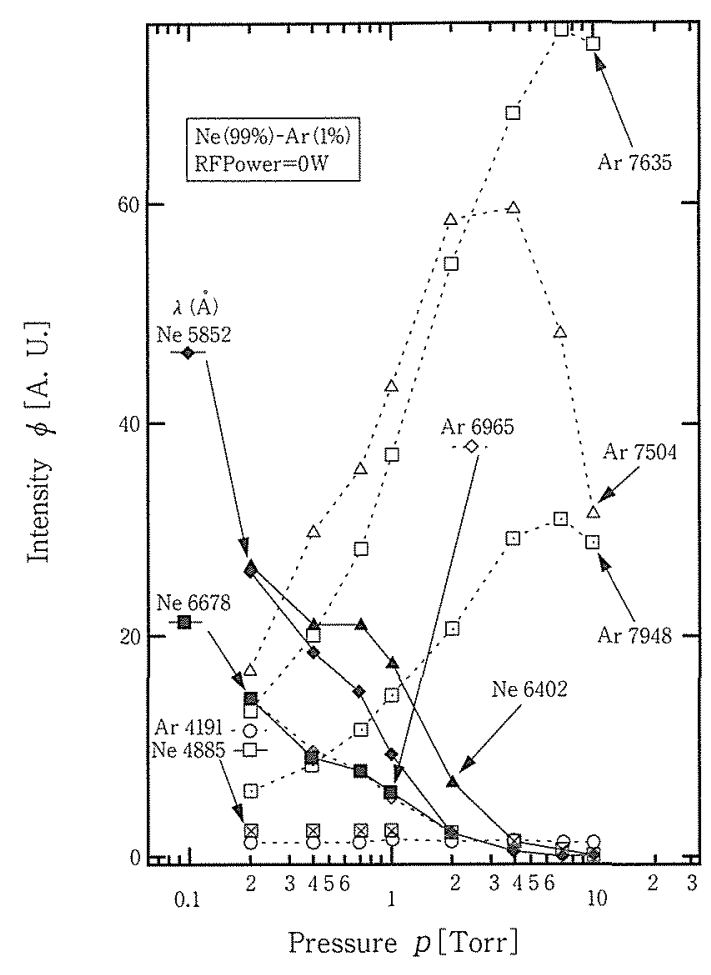

Fig. 3 (b)

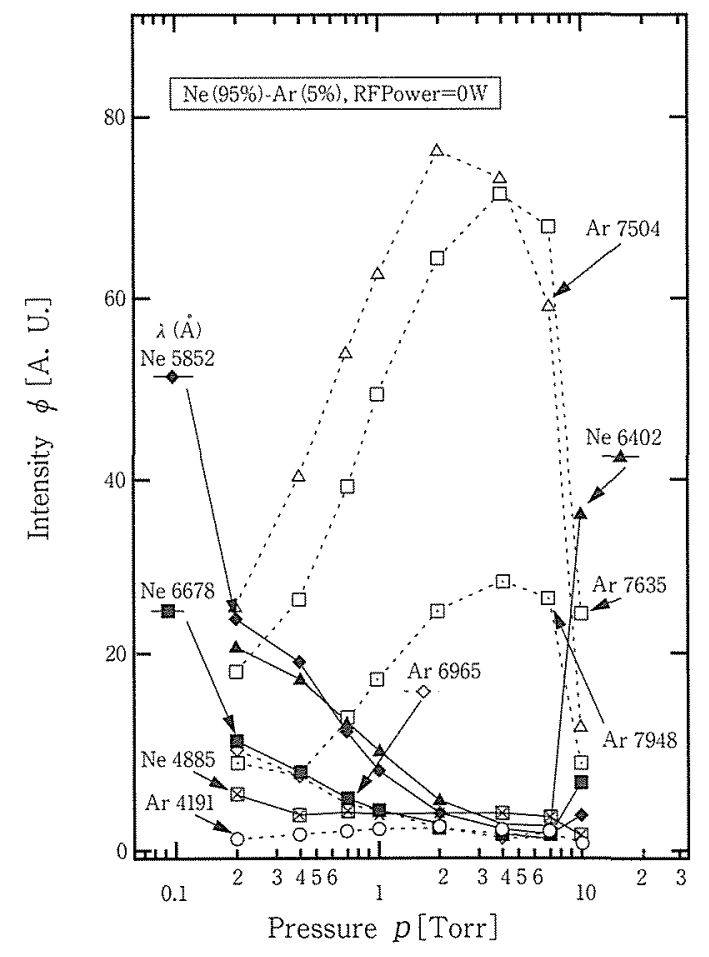

Fig. 3 (d) 


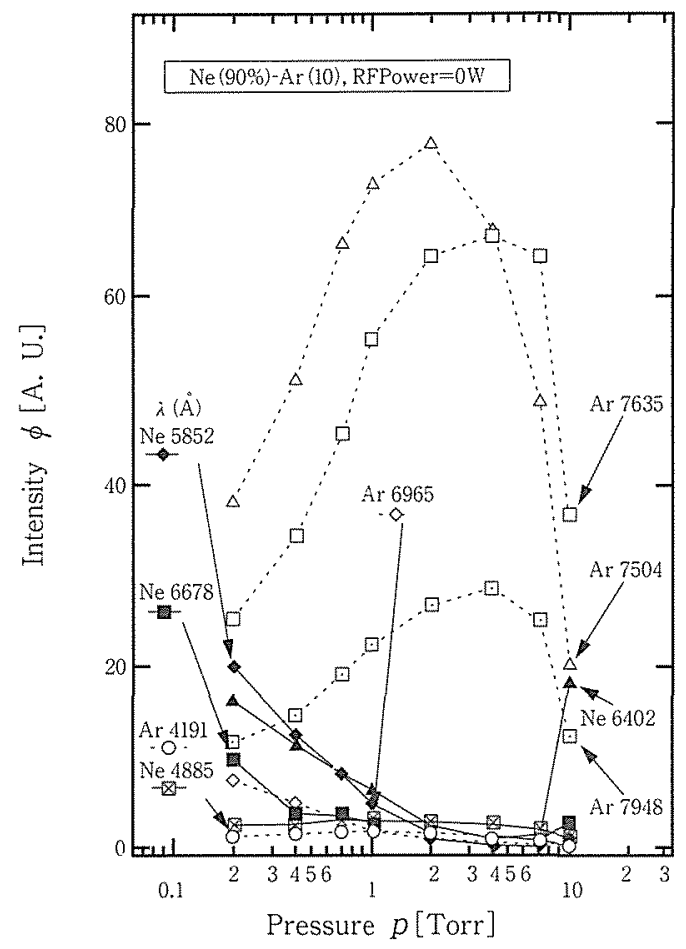

Fig. $3(e)$

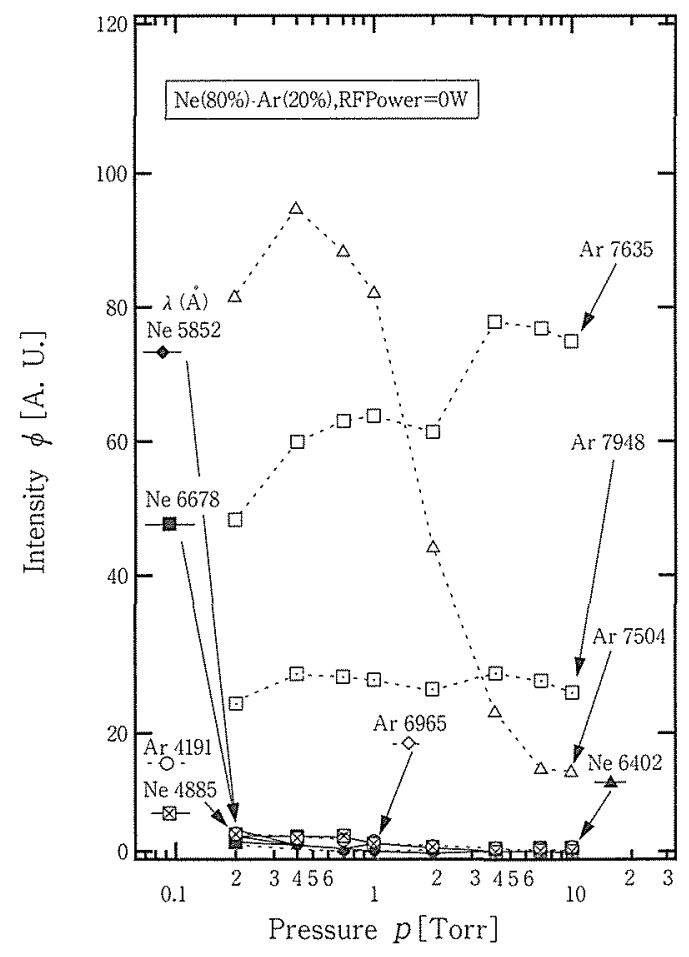

Fig. $3(f)$

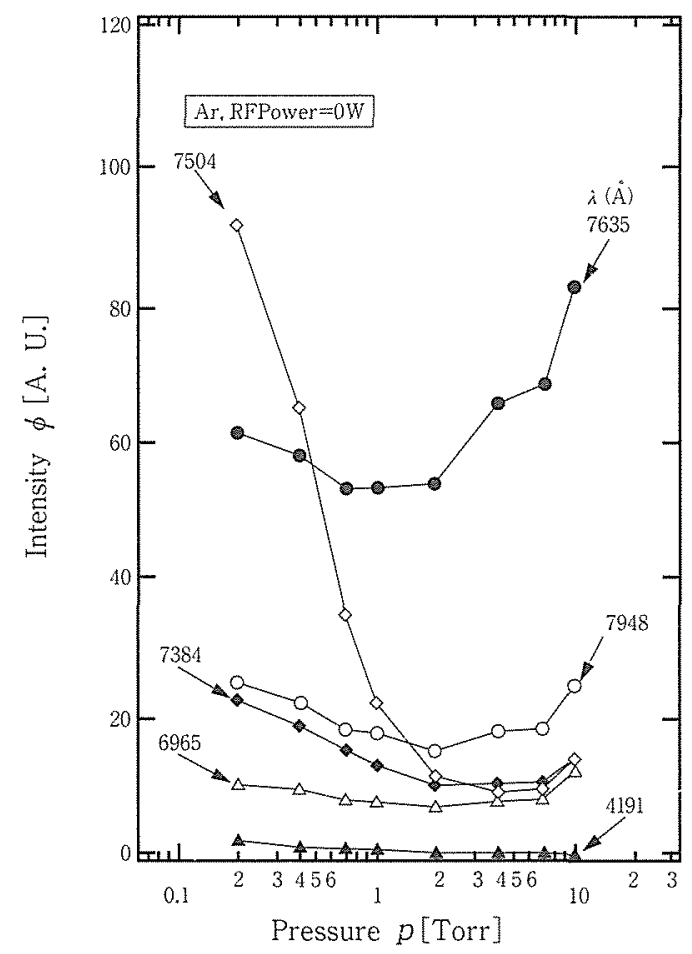

Fig. 3 (g)

Fig. 3: Spectral intensities of the spectral lines in the Ne-Ar gas mixtures as a function of gas pressure with a discharge current of $20 \mathrm{~mA}$ without RF powers. :
(a) Pure $\mathrm{Ne}$
(c) $\mathrm{Ne}(97 \%)-\mathrm{Ar}(3 \%)$
(b) $\mathrm{Ne}(99 \%)-\mathrm{Ar}(1 \%)$
(e) $\mathrm{Ne}(90 \%)-\mathrm{Ar} \quad(10 \%)$
(d) $\mathrm{Ne}(95 \%)-\mathrm{Ar} \quad(5 \%)$
(g) Pure $\mathrm{Ar}$
(f) $\mathrm{Ne}(80 \%)-\mathrm{Ar} \quad(20 \%)$ 


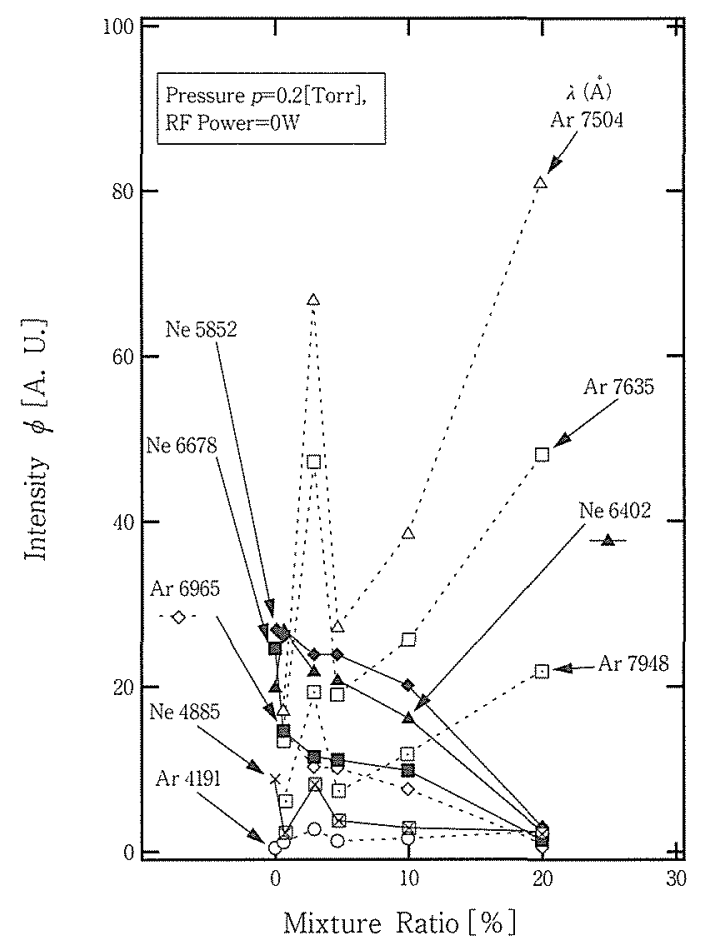

Fig. 4 (a)

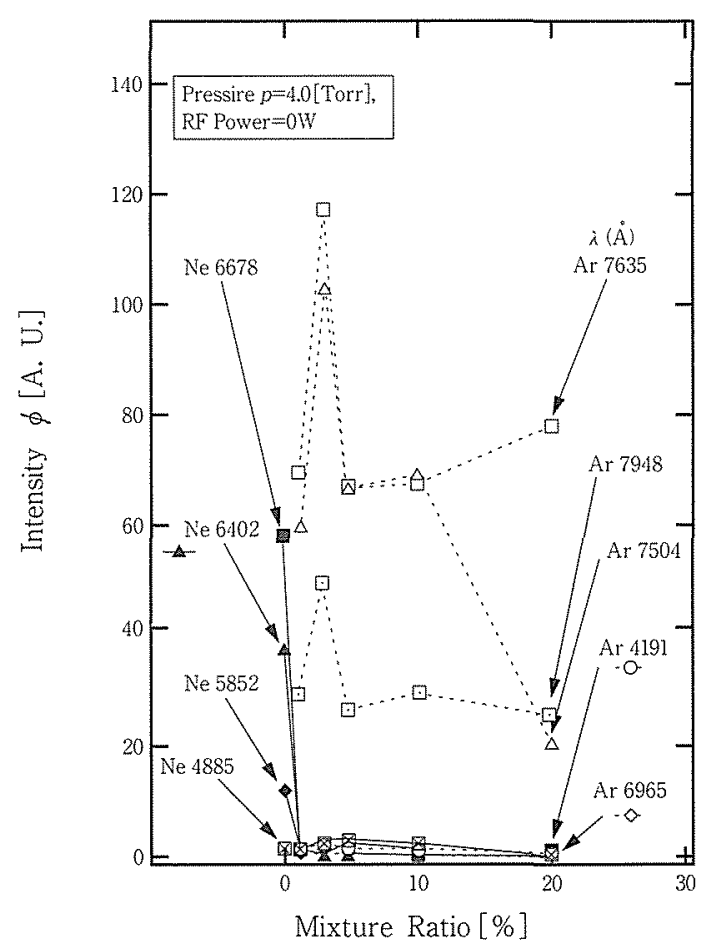

Fig. 4 (c)

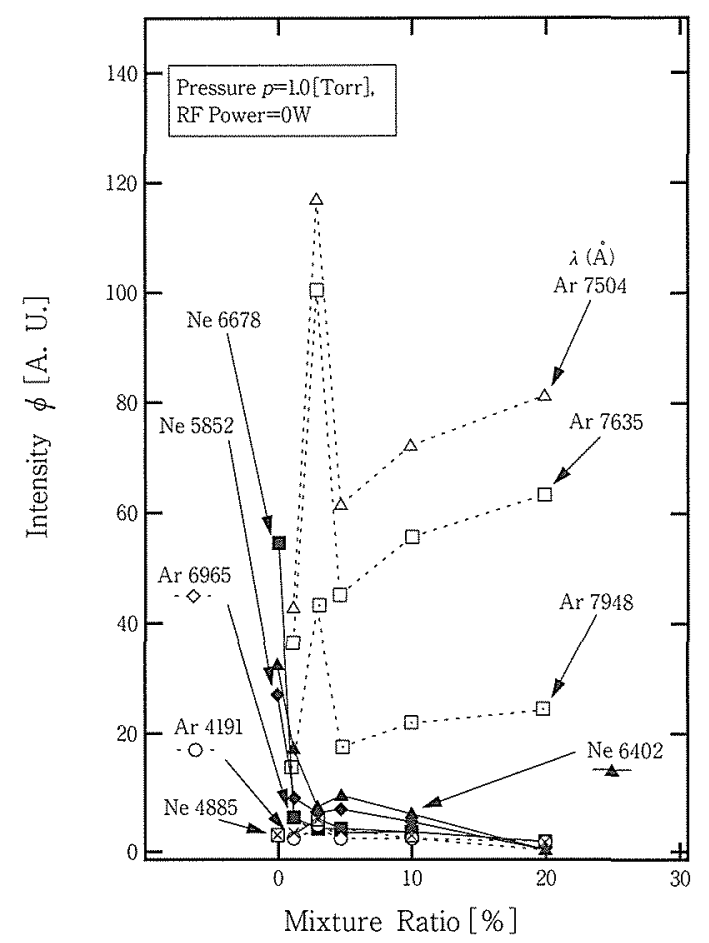

Fig. 4 (b)

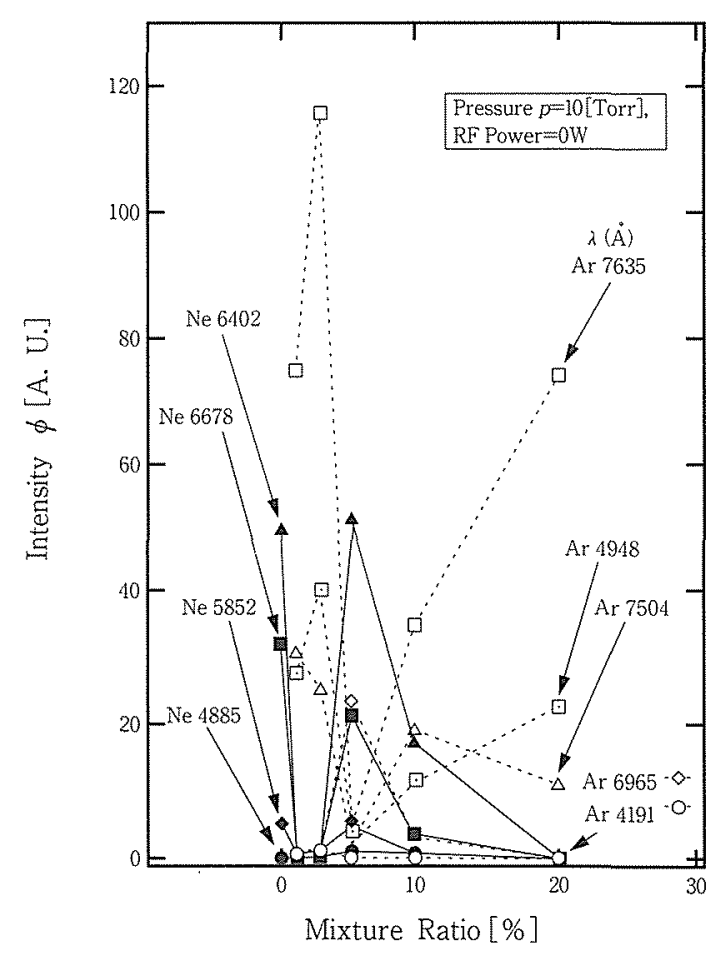

Fig. 4 (d)

Fig. 4: Spectral intensity of the spectral lines in the Ne-Ar gas mixtures without RF power as a function of the percentage of the mixture of argon of the total pressure with a discharge current of $20 \mathrm{~mA}$, and a gas pressure of:
(a) $p=0.2[$ Torr $]$
(b) $p=1.0[$ Torr $]$
(c) $p=4.0[$ Torr $]$
(d) $p=10$ [Torr] 


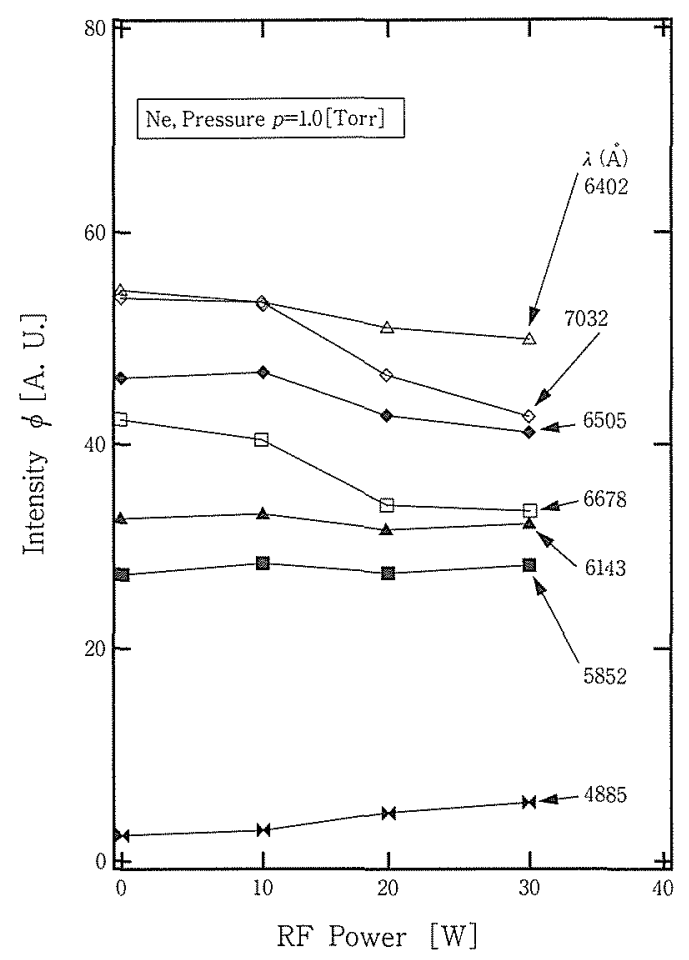

Fig. 5 (a)

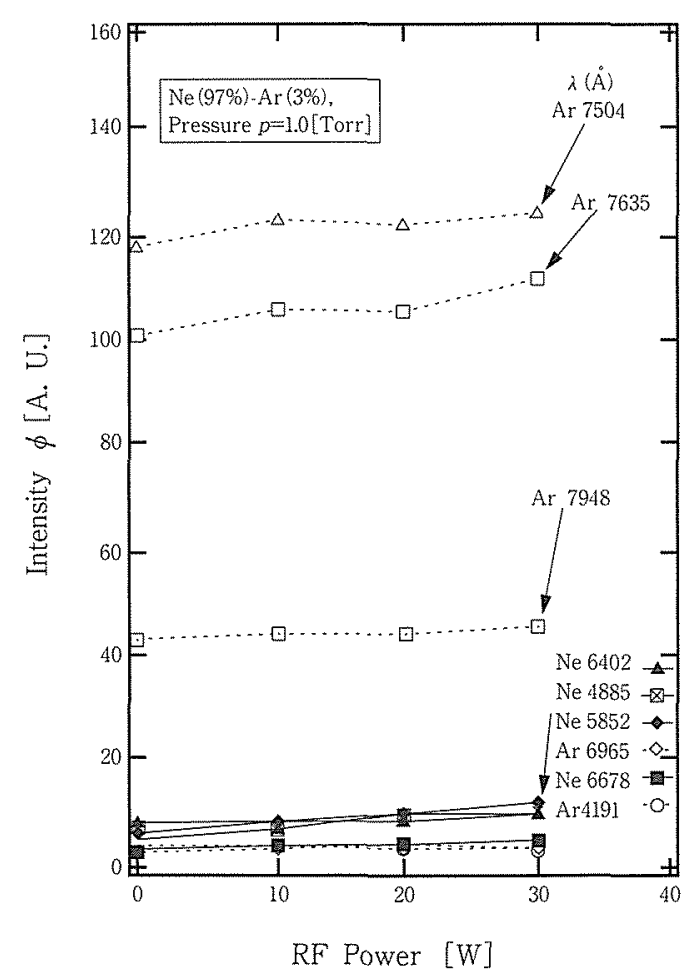

Fig. 5 (c)

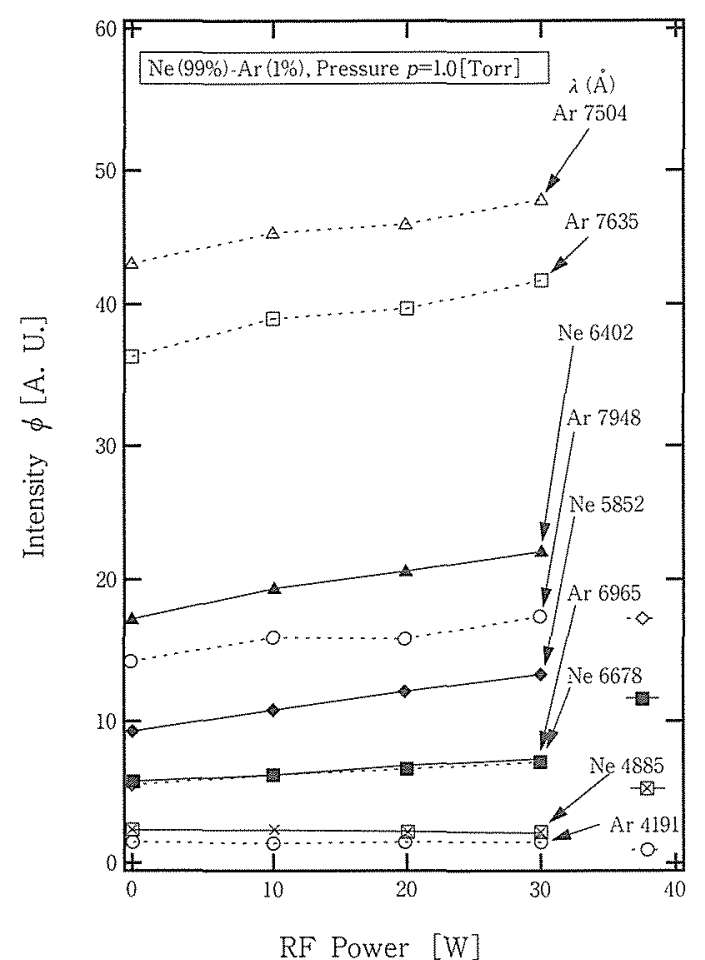

Fig. 5 (b)

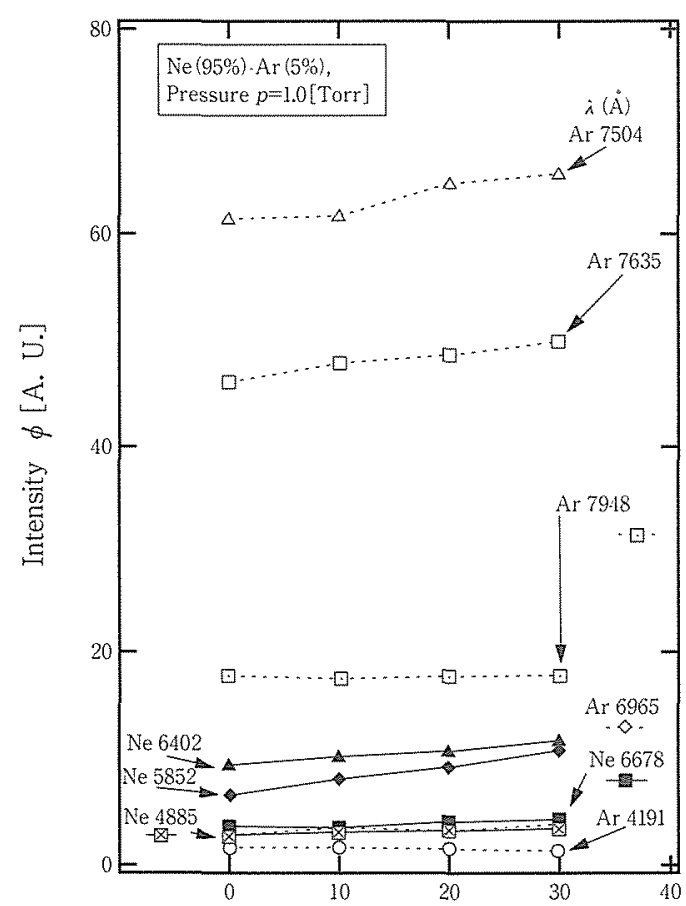

RF Power [W]

Fig. 5 (d) 


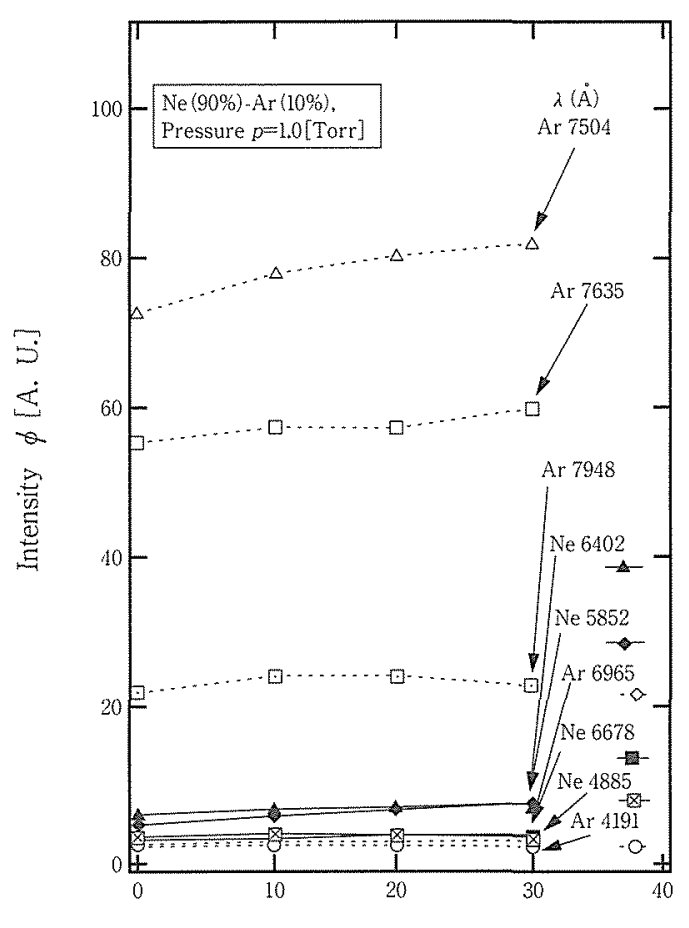

RF Power [W]

Fig. 5 (e)

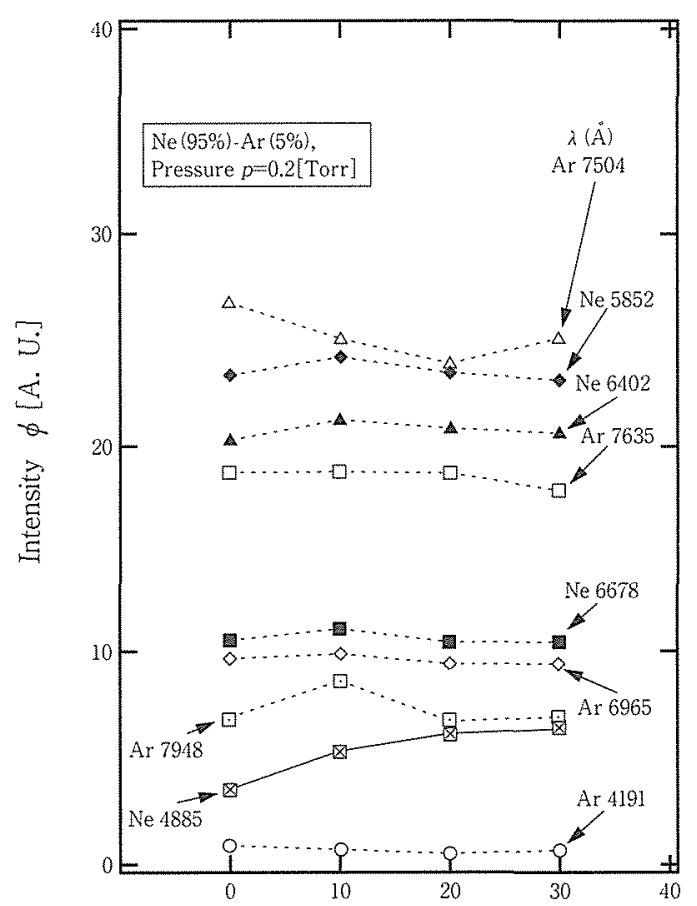

RF Power [W]

Fig. 5 (g)

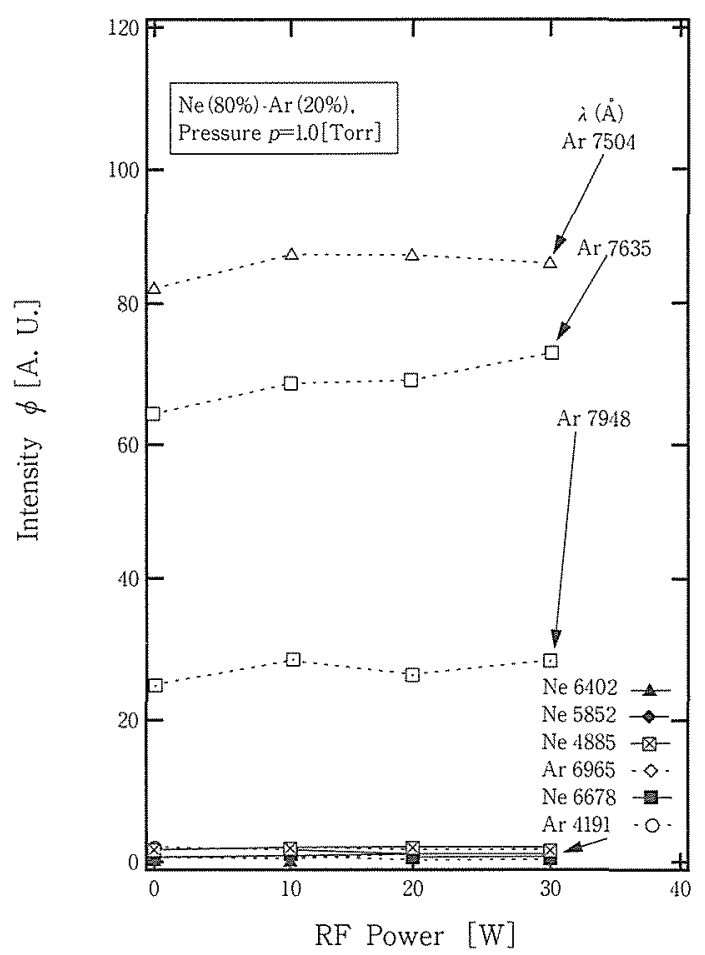

Fig. $5(f)$

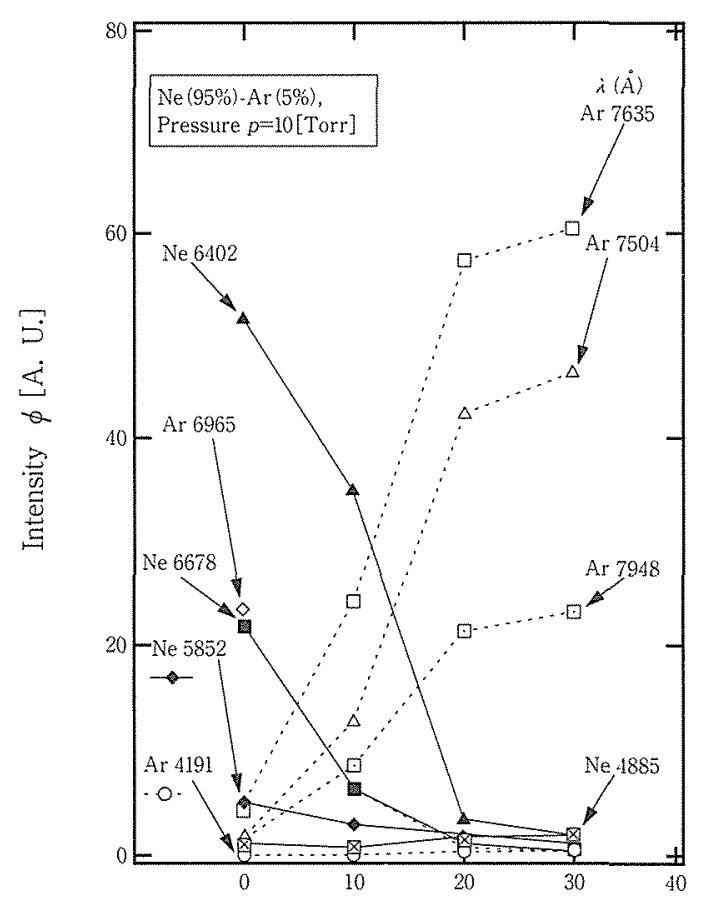

RF Power [W]

Fig. $5(h)$

Fig. 5: Spectral intensities of the spectral lines in the Ne-Ar gas mixture as a function of RF power with a discharge current of $20 \mathrm{~mA}$ for mixture ratio and gas pressure of:
(a) Pure $\mathrm{Ne}$,
$p=1.0[$ Torr $]$
(c) $\mathrm{Ne}(97 \%)-\operatorname{Ar}(3 \%)$
$p=1.0[$ Torr $]$
(b) $\mathrm{Ne}(99 \%)-\mathrm{Ar}(1 \%)$
$p=1.0[$ Torr $]$
(e) $\mathrm{Ne}(90 \%)-\operatorname{Ar}(10 \%) \quad p=1.0$ [Torr]
(d) $\mathrm{Ne}(95 \%)-\operatorname{Ar}(5 \%)$
$p=1.0[$ Torr $]$
(g) $\mathrm{Ne}(95 \%)-\operatorname{Ar}(5 \%) \quad p=1.0$ [Torr]
(f) $\mathrm{Ne}(80 \%)-\operatorname{Ar}(20 \%)$
$p=1.0[$ Torr $]$
(h) $\mathrm{Ne}(95 \%)-\operatorname{Ar}(5 \%) \quad p=1.0$ [Torr] 
sities of argon attain their maximum values at an argon mixing ratio of $3 \%$ and then remain somewhat constant expect for $\lambda=7504(\AA)$, which decreases with the increase in the mixing ratio.

Figure $4(\mathrm{~d})$ exhibits the spectral intensities at the pressure of $p=10$ Torr as a function of the gas mixing ratio. At this pressure, the spectral intensities for wavelengths of $\lambda=6402(\AA)$, and $6678(\AA)$ of neon, which weaken with increasing the gas pressure to 4.0 Torr as shown in Figs. $4(\mathrm{a})-4(\mathrm{c})$, are found to be strengthened again with the addition of $5 \%$ argon. After that, they decrease with increasing the mixing ratio. However, the spectral intensities for argon attain their maximum values at the argon mixing ratio of $3 \%$ and then decrease remarkably the minimum values at the mixing ratio of $5 \%$. After that, they increase again with increasing the mixing ratio of argon. These dependencies of spectrum on the gas mixing ratio are similar to those observed in Figs. $4(\mathrm{a})-4(\mathrm{c})$.

Figure 5 examines the spectral intensity as a function of the RF power superimposed on the discharge tube. In Fig. 5 (a), the normalized spectral intensities for pure neon are shown at $p=1.0$ Torr as a function of the superimposed $\mathrm{RF}$ power. Here, the spectral intensities for wavelengths $\lambda=6402,7032$, and $6678(\AA)$ decrease with increasing RF power, while only the spectral intensity of $\lambda=4885$ $(\AA)$ increases. The intensities of wavelengths $\lambda=5852$ and $6143 \mathrm{~A}$ remain almost constant, having no dependence on RF power at this pressure.

In Fig. 5 (b), all wavelengths exhibit similar characteristics for the argon mixing ratio of $1 \%$, with almost all spectral intensities of neon and argon increasing with increasing RF power. In Figs. 5 (c), (d), (e), and (f), similar characteristics are found for argon mixture ratios of $3 \%, 5 \%, 10 \%$ and $20 \%$, respectively. It is concluded that the RF power has little influence on the spectral intensities at this pressure (1 Torr).

In Fig. $5(\mathrm{~g})$, the normalized spectral intensities for the argon mixing ratio of $5 \%$ are shown for $p=0.2$ Torr as a function of RF power. As shown in this figure, only the neon spectral intensity of $\lambda=4885(\AA)$ increases over the entire range of $\mathrm{RF}$ power, while $\lambda=7504(\AA)$ of argon decreases when RF power increases from zero to $20 \mathrm{~W}$, and then increases.

In Fig. 5(h), the influence of the RF power on the spectral intensities at a mixing ratio of $5 \%$ is shown for gas pressure of 10 Torr. As shown in this figure, all the neon spectral lines sharply decrease with increasing RF power. On the contrary, the spectral lines of argon exhibit a considerable increase as the RF power is increased.

\section{Discussion}

The radiation emitted from the positive column in a glow discharge tube occurs when an excited electron in gas atom is transferred from an upper excited energy level to a lower level. The excited gas atoms are usually produced by the collision with electrons having an energy higher than the excited energy level. The number of these electrons approximately depends on the electron density and the electron temperature. Usually, the electron density increases as the gas pressure increases when the discharge current is held constant. This tendency results from the fact that the electron mobility decreases with increasing gas pressure due to the collisions among electrons and gas atoms increasing with gas pressure. Meanwhile, the electron temperature decreases with an increase in gas pressure as shown by Schottky's ambipolar diffusion theory ${ }^{(8)}$. Therefore, over the range of lower gas pressures, the tendency of the increase in radiation with increasing gas pressure is mainly due to the increase in the electron density. After reaching the maximum value of radiation at a given pressure, the decrease in radiation is caused by the decrease in the electron temperature with increasing gas pressure.

As shown in Fig. 3(a), it was found that almost all spectral intensities, except for $\lambda=4885$ and $5852(\AA)$, sharply increase as the gas pressure changes from 0.2 to 1.0 Torr. This dependence on pressure is partly caused by the electron density as related to the gas pressure as described above.

Moreover, this characteristic is strengthen by the effect of the stepwise excitation of neon gas atoms from the metastable state. In neon gas, the levels of the metastable state are 16.72 and $16.62 \mathrm{eV}$, as shown in Fig. 6. The number of metastable state gas atoms is usually proportional to the electron density, and therefore, increases with gas pressure. The stepwise excitation from the metastable state to upper energy levels easily occurs through the collisions with the electrons rather than by the direct excitation from the ground state.

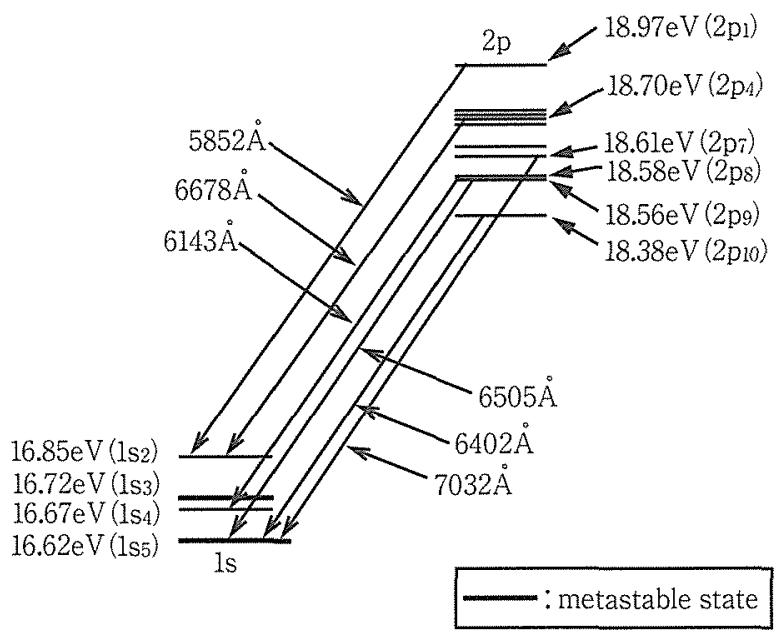

Grotrian Diagram for Neon I

Fig. 6 : Grotrian diagram for neon.

The difference between the energy level of the metastable state and the upper level is several electron volts, as shown in Fig. 6. Here, it is found that the levels are separated into two energy level groups. The first group related to the lower energy levels (Is) and contains the metastable state levels, while the second group corresponds to higher energy levels (2p). It can be easily calculated that the average energy difference between these two groups is only of the order of several electron volts, as shown in Table. 1.

The electron temperature in a rectangular discharge tube of a similar size has been measured with the use of electric 
Table. 1: Energy difference for each wavelenght having lower upper energy levels.

\begin{tabular}{|c|c|c|}
\hline Wavelength $[\AA]$ & Lower-Upper Levels & Energy Diference $[\mathrm{eV}]$ \\
\hline 5852 & $1 \mathrm{~s}^{2}-2 \mathrm{p} 1$ & 2.12 \\
\hline 6143 & $1 \mathrm{~s}_{5}-2 \mathrm{p} 7$ & 1.99 \\
\hline 6402 & $1 \mathrm{~s}_{5}-2 \mathrm{p} 9$ & 1.94 \\
\hline 6505 & $1 \mathrm{~s}_{4}-2 \mathrm{p} 8$ & 1.91 \\
\hline 6678 & $1 \mathrm{~s} 2-2 \mathrm{p} 4$ & 1.85 \\
\hline 7032 & $1 \mathrm{~s}_{5}-2 \mathrm{p} 10$ & 1.76 \\
\hline
\end{tabular}

probes by T. Kaneda ${ }^{(11)}$. From this result, it was found that the electron temperature decreases from $4.0 \mathrm{eV}$ to $2.6 \mathrm{eV}$ as the gas pressure increased from 0.2 to 1.0 Torr. This means that the most probable electron energy decreased from approximately $4.0 \mathrm{eV}$ to $2.6 \mathrm{eV}$, if the electron energy distribution was Maxwellian. Therefore, the probability of the stepwise excitation from the metastable energy level state increased as the gas pressure was increased from 0.2 to 1.0 Torr. This was due to the collision cross section of stepwise excitation having a resonance maximum value with the energy differences of the stepwise excitation. As a result, the decrease of the electron temperature with increasing gas pressure effectively raised the stepwise excitation, and likewise increased the spectral intensity as the gas pressure increased.

As shown in Fig. 3 (b), the spectral intensity of neon decreased as the gas pressure increased when $1 \%$ argon was added to the neon. The spectral intensity of argon considerably increased with increasing gas pressure and then decreased after reaching the maximum value. These spectral characteristics are caused by the Penning effect that the neon gas atoms in the metastable state collide with argon gas atoms, and in the process ionizes them. As indicated with pure neon gas, the density of neon in the metastable state is increased as the gas pressure increased. The atoms are used for the production of electrons by the ionization of argon gas instead of being used for the stepwise excitation of neon gas. As a result, the electron density is increased more efficiently by this effect, in addition to that due to the increase in gas pressure. As shown for neon gas, these electrons produce the metastable state argon gas atoms.

The energy levels of argon gas are shown in Fig. 7. From this figure, it is seen that the stepwise excitation can be easily produced by the lower energy electrons and within the excited argon gas atoms and through this effect, radiation is emitted. Taking into account the total of these effects, the spectral characteristics of argon are also exhibited as shown in this figure.

The phenomenon that the spectral intensity of argon is enhanced by the Penning effect can be observed more readily as shown in Fig. $3(\mathrm{c})$, where the mixing ratio of argon attains $3 \%$. However, the spectral intensity of argon decreases when the ratio is increased to values greater than $5 \%$. When the mixing ratio of argon is over $20 \%$, the spectral intensity lines observed are almost exclusively argon.

As shown in Fig. 4 (a)-Fig. 4 (d), it was also observed that overall the spectral intensities of argon, after reaching relative minimums at an argon mixing ratio of $5 \%$, markedly increased when the ratio was further increased. The spectral intensities were shown as a function of the mixing ratio

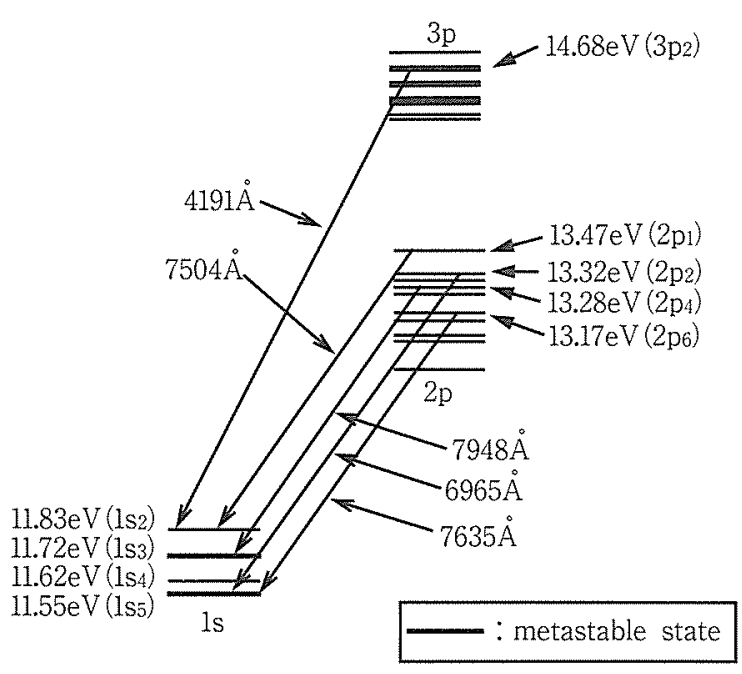

Grotrian Diagram for Argon I

Fig. 7 : Grotrian diagram for argon.

when the gas pressure was held at 0.2 Torr (Fig. 4 (a)). In this figure, the spectral intensities of argon were considerably increased when the mixture ratio of argon attained $3 \%$. These peak characteristics are caused by the Penning effect as described above. It was also observed that overall the argon spectral intensity showed a remarkable increase after the intensity fell to the minimum at the $5 \%$ ratio of argon. This effect is mainly caused by the increase in the argon partial pressure, which increases with the rise of the argon mixing ratio. The electrons collide with argon gas atoms more effectively, exciting them as the mixing ratio of argon increases. This is turn increased the spectral intensity of argon. While, the spectral intensities of neon decrease with the argon mixing ratio. This phenomenon is inverse effect of that for argon because the neon partial pressure decreases with the rise of the argon mixing ratio.

As shown in Fig. $4(\mathrm{~d})$, the spectral intensities of neon gas, which decrease with increasing argon mixing ratio and almost disappear at the gas pressure of 4 Torr (Fig.4 (c)), appear again suddenly at the gas pressure of 10 Torr. It is observed that the spectral intensities of neon gas have maximum values at the mixing ratio of $5 \%$ argon. This phenomenon corresponds to those observed in Fig. 3 (d) and Fig. $3(\mathrm{e})$, where the spectral intensities of neon gas rise abruptly at the gas pressure of 10 Torr. It is noticed that this distinctive phenomenon occurs at the discharge condition that the gas pressure is extremely high value such as 10 Torr and that the mixing ratio of argon is also comparative high values of $5 \%$ and $10 \%$.

It is expected that the molecular ions $\mathrm{Ne}_{2}{ }^{*}$ can be produced in such a high gas pressure by the following collision process;

$$
\mathrm{Ne}^{+}+2 \mathrm{Ne} \rightarrow \mathrm{Ne}_{2}^{+}+\mathrm{Ne}
$$

These molecular ions of neon tend to make a dissociative recombination with electrons as the electron temperature decrease, for the dissociative recombination coefficient, which is much higher value than the radiative coefficient, increases as the electron temperature decreases ${ }^{(15)}$. Therefore, it is 
expected that the molecular ions of neon would be produced at the gas pressure of 10 Torr. They make the dissociative recombination with electrons at the condition that the electron temperature decreases by the addition of argon at the mixing ratio of $5 \%$ as follow.

$$
\mathrm{Ne}_{2}{ }^{+}+e \rightarrow 2 \mathrm{Ne}+\varepsilon
$$

The excess potential energy $\varepsilon$ at this collision process is used for the excitation of neon gas. Therefore, it is observed that the spectral intensities of neon gas appear again in the extensive discharge condition.

The pure neon spectral intensities at the pressure of 1.0 Torr were shown as a function of superimposed RF power (Fig. 5(a)). It was found that the spectral intensities were only slightly influenced by the RF power. The dependences of the spectral intensities on the RF power for various argon $/$ neon gas mixtures under the gas pressure of 1 Torr were also examined (Figs.5 (b) - 5 (f)). It was found that the spectral intensities of neon decreased as the argon mixing ratio increased and that the $R F$ power had only a slight influence on the spectral intensities at this gas pressure. It has generally been expected that the electron density and the electron temperature would be influenced by the RF power since the collision of electrons with the gas atoms should be dependent on the RF power. However, large influences on the spectral intensities were not found at this gas pressure.

On the other hand, when the mixing ratio of argon was $5 \%$ at the gas pressure of 10 Torr (Fig. $5(\mathrm{~h})$ ), the spectral intensities of neon decrease as the RF power increased while conversely, those of argon increased considerably. The ambipolar diffusion theory in the positive column states that the electron temperature decreases with increasing gas pressure. Meanwhile, the electron density increased with increased gas pressure due to the decrease in electron mobility. Therefore, it is expected that the electron temperature at the gas pressure of 10 Torr will be considerably lower compared with those at the gas pressure of 1.0 Torr, while the electron densities at 10 Torr will be higher comparing with that at 1.0 Torr. Moreover, as is described in the explanation about Fig. 4 (d), the molecular ions of neon gas are produced in this discharge condition. As a result, the dissociative recombination between the neon molecular ions and electrons at 10 Torr highly occur comparing with that for the lower gas pressure. Therefore, the spectral lines of neon are observed extensively at this discharge condition when the RF power is not applied.

Under this discharge condition, when the RF power is applied to the positive column, the electron temperature and the electron density would be increased. By increasing the electron temperature, the rate of the dissociative recombination would be decreased. Therefore, the spectral lines of neon decrease considerably with increasing the applied RF power.

On the other hand, the increases of electron temperature and electron density increase the number of metastable state neon gas atom. These increased numbers of metastable state neon gas atoms collide with argon gas atoms, exciting and ionizing them by the Penning effect. This phenomenon occurs more effectively at higher RF power. Then the spectral intensities of argon increase considerably with increasing RF power.

These results are very interesting because the spectral intensities are selected or controlled by $\mathrm{RF}$ power.

\section{Conclusion}

The spectral intensities emitted from a positive column in a rectangular discharge tube were measured as functions of the gas pressure, mixing ratio of argon/ neon, and with and without superimposed RF power.

Almost all spectral lines of pure neon attain maximum characteristics, while those of pure argon display minimum characteristic with an increase in gas pressure. When a small amount of argon gas is added to the neon gas, the neon spectrum decreases with increasing gas pressure. On the other hand, the argon spectrum displays a marked increase with increasing gas pressure. This tendency becomes stronger with the addition of more argon gas. At a mixing ratio of argon of $3 \%$, the spectral intensities of argon markedly increase due to the Penning effect.

The behaviors of the spectral intensities when the positive column is subjected to an RF power were also studied as a function of the RF power. The spectral intensities exhibited only slight changes at the gas pressure of 1.0 Torr when the mixing ratio of argon increased from $1 \%$ to $20 \%$. However, the spectral intensities were found to be greatly influenced by the RF power when the gas pressure was 10 Torr. Under these conditions, the spectral intensity of neon decreased while those of argon increased as the RF power was increased.

\section{References}

(1) Chang, J.S. Ichikawa, Y. and Teii, S. : "The effect of discharge tube shape and flow velocity on the electron temperature of a medium pressure positive column.", Jpn. J. Appl. Phys., 18 (1979) 847-848.

(2) Wilhelm, H. E. : Cdn. J. Phys. 50 (1972) 1156-1160.

(3) Kaneda, T. Imamoto, N. Kubota, T. and Chang, J. S. : "The electron temperature in a medium pressure rectangular tube positive column plasma.", Bull. Am. Phys. Soc., 29 (1984) 143.

(4) Kaneda, T.Kubota, T. and Chang, J.S. : "Electron temperature and axial electric field in a medium pressure $\mathrm{He}-\mathrm{Ne}$ and He-Ar gas mixture rectangular tube positive column plasma.", 17th ICPIG Conf., 2 (1985) 691-693.

(5) Chang, J. S. : "The electron temperature in a positive column with volume recombination", J. Phys. D : Appl. Phys., 9 (1978) L137.

(6) Abrams, R. L. : "Gigahertz tunable wave guide $\mathrm{CO}_{2}$ laser.", Appl. Phys. Lett., 25 (1974) 304-306.

(7) Yamamoto, K. Chang, J. S. Matsumura, S. and Teii, S. : 8th Int. Symp. Plasma Chem., 3 (1987) 1279.

(8) Schottky, W. : "Diffusion Theorie der positiven Säule.", Phys. Zeits., 25 (1924) 635-640.

(9) Kaneda, T. Ohsone, Y. and Chang, J. S. : "Plasma parameters in a rectangular tube positive column neon plasma.", Jpn. J. Appl. Phys., 29 (1990) 1324-1328.

(10) Kaneda, T. : "The characteristics of a positive column plasma 
by the AC transverse magnetic field.", J. Light \& Vis. Env., 14 No, 1 (1990) 8-14.

(11) Kaneda, T.: "The characteristics of a positive column in a rectangular discharge tube.", J.Light \& Vis. Env. 12 (1988) $64-71$.

(12) Kaneda, T.: "The optical characteristics of a positive column gas mixture plasma in a capillary discharge tube.", J. Light \& Vis. Env., 14 No, 2 (1990) 1-10.

(13) Kaneda, T. Kubota, T. Ohuchi, M. and Chang, J. S. : "Timeaveraged electric field profiles in a capacitive coupling parallel plate electrode RF discharge helium plasma.", Jpn. J. Appl. Phys., 29 (1990) 2497-2498.

(14) Kaneda, T. Kubota, T. Ohuchi, M. and Chang, J. S. : "Timeaveraged electric potential profiles in a capacitive-coupling parallel-plate electrode neon gas RF discharge plasma." J. Phys D : Appl. Phys., 23 (1990) 1642-1647.

(15) Chang, J. S. Hobson, R. M. Ichikawa, Y. Kaneda, T. Maruyama, $\mathrm{N}$.and Teii, S : "Dissociative recombination of $\mathrm{Ne}_{2}{ }^{+}$at elevated electron and gas temperatures." J. Phys. B : At. Mol. Opt. Phys., 22 (1989) L665-L668. 